\title{
Production of functional materials on the basis of tungsten multicomponent mineral resources
}

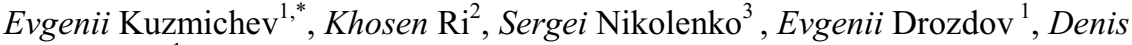 \\ Balakhonov $^{1}$ \\ ${ }^{1}$ Far Eastern State Transport University, Seryshev Str., 47, Khabarovsk, 680021, Russia \\ ${ }^{2}$ Pacific State University, Tikhookeanskaya Str., 136, Khabarovsk, 680035, Russia \\ ${ }^{3}$ Russian Academy of Sciences, Tikhookeanskaya Str., 153, Khabarovsk, 680035, Russia
}

\begin{abstract}
We produced tungsten and tungsten carbide as a result of the synthesis of multicomponent tungsten mineral resource with the employment of high density energy flow. The results of experiments concerned with the possibility of using high-density energy to synthesize functional materials for industrial use are presented as well.
\end{abstract}

\section{Introduction}

The growing difficulties in supply of raw materials and the high cost of expansion of steel production require attention to the problem of the use of resource-saving technologies in all sectors. The objective is to obtain the maximum number of higher quality and low-cost steel products from a certain amount of source raw material.

These problems have set a series of urgent tasks and identified strategic directions for materials science in the fields of development and production of materials:

- production of materials involving complex use of raw materials and in consideration of their distribution in nature;

- design and development of innovative, environmentally friendly technologies for the production of structural, instrumental, welding and surfacing materials from mineral raw materials without deep technological processing and locating these technologies closer to the production sites and mining areas.

Cyclic circulation of substances and materials can be considered as the basis for the resolution for the problem of producing new materials. Such approach was first proposed within the framework of the concept of sustainable development as the transition to a fundamentally new technology, allowing the society to fit into the "endless circle" of natural processes on the planet, is the only possible way of the radical improvement of the whole spectrum of interaction between the society and the environment [1]. The so-called "cycle of materials" has been studied in Germany for more than 10 years [2]. At the same time there is a significant impact of the new approach on the formation of a new unifying science of materials - "Integral Material Science". This means that the "life" of all groups

\footnotetext{
*Corresponding author: e_kuzmichev@,mail.ru
} 
of materials in use as a means of human activity is traced "from nucleation to death" and their further reuse in the form of other means of action.

This approach based on the environmental safety is of great importance for the future.

Analysis of dynamics in the initial stages of substances and materials (Geology Mining - Metallurgy - Materials Science) shows that efficiency of the materials production increases if the distance between the cycles and cycle time is minimal.

Therefore, sustainable development of the region requires organization of processing minerals at their mining areas using high technologies for processing raw materials under environmentally sound conditions, which contributes to the minimum increase in entropy within the framework of cycle and between cycles [3].

A number of studies $[2,4]$ indicate the prospects of using concentrated energy flows to obtain materials and products from mineral raw materials in their mining areas. The research is based on the multipurpose use of mineral raw materials when exposed to concentrated energy flows, in order to extract the alloying elements and oxides. This fact corresponds to the proposed theory of cross-entropy minimization when producing final material, leaving aside the stages of the primary beneficiating and reprocessing. Reducing the production period of material is possible by use of modern technologies, which exclude the use of environmentally hazardous pyro- and hydrometallurgical processes. The use of such technologies will not only ensure complex and rational processing of mineral resources, but also the compliance with environmental requirements and transformation of mining regions dealing with extracting resources into developed suppliers of materials and products.

Tungsten containing mineral raw material is a rebellious complex difficult for concentration, and the method of its decomposition includes complex processes of reprocessing and preparation. According to the ways of processing tungsten containing raw materials two groups of methods are distinguished: hydrometallurgical and thermochemical.

Among the hydrometallurgical methods of processing raw materials, the lion's share belongs to their decomposition with mineral acids or alkalis at normal or high-top pressure. The most common way of breaking-down mineral raw materials is the use of toxic chemicals: hydrochloric, sulfuric and nitric acids [1,3], but this causes significant environmental damage. Upon an exploitation of an ore deposit up to $5 \%$ in average is considered to be the shipping ore, up to $35 \%$ is concentrated and up to $60 \%$ of base ore is left in the ore deposit [5]. Only $2 \%$ of mined rock turns into useful products and $98 \%$ is lost in the waste, and the biggest losses occur at the stages of concentration and technological conversion.

The method, that has found its use in the practice of processing scheelite concentrates, is conducted using sodium hydroxide solution. Complete decomposition is achieved in the processing of finely divided concentrate of $25-40 \%$ sodium hydroxide solution at $110-120^{\circ}$ $\mathrm{C}$, and the excess alkali makes $50 \%$ or more. The process is not applicable to low grade concentrates, and self-decomposition takes a long time.

Method of autoclave-soda leaching, developed in the USSR, is widespread these days. This method is one of the most common in the hydrometallurgy of tungsten on the decomposition of tungsten products, and is noted in numerous studies $[4,5,6]$ on autoclavesoda process of decomposing. But along with its benefits we can also point to its significant disadvantages: high consumption of soda, insufficiently high degree of decomposition in a single process stage and considerable consumption of hydrochloric acid for neutralization of solutions as well as pressure operated devices. An alternative to this decomposition method is hydrothermal treatment with alkali solutions at moderate temperatures (150-170 ${ }^{\circ} \mathrm{C}$ ) in order to reduce the working pressure (4-6 atm) to intensify leaching process and 
significantly reduce the consumption of an alkaline reagent and energy for technological needs [6].

The method based on soda sintering of tungsten concentrates at temperature of about $850-880{ }^{\circ} \mathrm{C}$ ensures $90-93 \%$ transformation of tungsten into water soluble tungsten compound, according to works [7-10], describing the method of preparation of the charge in the furnace in the way that it does not melt but takes the form of lumps. Such result can be achieved by adding the leaching tailings of sintered mass in the amount that is sufficient to reduce $\mathrm{WO}_{3}$ content up to $20-22 \%$. The break-down degree reaches $99-99.5 \% \mathrm{WO}_{3}$. There also exist several processes that were described [11] and have practical use. But all these processes have a multi-stage structure with a large share of use of various kinds of chemical raw materials, acids, alkalis, etc. All of the above described methods have a detrimental effect on the environment, soil and rivers. One of the trends towards the creation of tungsten ore processing technologies that meet modern requirements is the transition to the processes employing highly concentrated energy flows, such as lowtemperature plasma technology and laser technology $[12,13]$.

The goal of our research was to produce tungsten carbide from scheelite concentrate by using direct-action plasma arc.

\section{Methods and materials}

Phase analysis of the fusion products was performed by the scanning electron microscope «VEGA $3 \mathrm{LMH»} \mathrm{(TESCAN),} \mathrm{equipped} \mathrm{with} \mathrm{energy} \mathrm{dispersive} \mathrm{spectrometer} \mathrm{«X-Max} \mathrm{80»}$ (Oxford Instruments). Photographing samples and micro-search was conducted mainly in the mode of backscattered electrons (BSE-detector). In the image obtained, phases with higher average atomic number are reflected in the contrast more vividly in comparison with the phases with lower average atomic number.

Phase analysis was also carried out using a ZEISS Libra-120 transmission electron microscope equipped with a detector and HAADF- $\Omega$-energy filter. Samples were examined in the modes of transmitted light, dark field and electron microdiffraction. The samples for transmission electron microscopy were prepared by electrolytic polishing and ion etching.

The compositions of the slag and the synthesized product were examined with diffractometer "Dron 7" with a tube of Co on the X-ray $K_{\alpha}$-line.

Exposure of refractory materials to high-density energy flows in the range of $g>10^{4}$ $10^{5} \mathrm{~W} / \mathrm{cm}^{2}, \mathrm{~g}>10^{6}-10^{8} \mathrm{~W} / \mathrm{cm}^{2}$ leads to molecular destructuring of the crystal lattice and the subsequent formation of a new one. This process causes the formation of materials with both mechanical and chemical properties changed. This can be used with some success in various technological processes, such as welding, overlay welding on the surface of machine parts subjected to heavy wear during service.

The use of high-temperature methods of impact on the mineral raw material of refractory origin and consequences of this impact are understudied and thus allow us not only to develop new methods of producing various functional materials based on mineral resources, but also to create new materials with a wide range of necessary properties, different from former ones produced by conventional methods. Such methods as plasma can solve the problem of changes in the properties of the workpiece surface. Plasma flow will deliver a certain chemical element in the crystal lattice of the metal. This chemical element processes the properties needed to create the desired effect, like increase in strength or endurance, preserving the integrity of one-piece structural elements, and increase in service life $[14,15]$.

For this reason, our task is to find alternative ways to produce tungsten and tungstencontaining compounds from tungsten containing raw materials using the above mentioned methods. As a material, we took CaWO4 scheelite concentrate with up to $30-55 \%$ 
proportion of tungsten against other elements. The chemical composition of "Lermontov Mining and Concentrating Mill" scheelite concentrate is presented in the Table. 1.

Table 1. Chemical composition of scheelite concentrate of the "Lermontov Mining and Concentrating Mill".

\begin{tabular}{|c|c|c|c|c|c|c|c|c|}
\hline \multicolumn{8}{|c|}{ The content of components in the concentrate, W \% } \\
\hline $\mathrm{SiO}_{2}$ & $\mathrm{Al}_{2} \mathrm{O}_{3}$ & $\mathrm{Fe}_{2} \mathrm{O}_{3}$ & $\mathrm{FeO}$ & $\mathrm{MnO}$ & $\mathrm{CaO}$ & $\mathrm{MgO}$ & $\mathrm{Na}_{2} \mathrm{O}$ & $\mathrm{K}_{2} \mathrm{O}$ \\
\hline 7.96 & 0.78 & 5.29 & 0.72 & 0.02 & 19.8 & 2.45 & 0.18 & 0.17 \\
\hline $\mathrm{P}_{2} \mathrm{O}_{5}$ & $\mathrm{As}$ & $\mathrm{TiO}_{2}$ & $\mathrm{WO}_{3}$ & $\mathrm{SO}_{3}$ & $\mathrm{H}_{2} \mathrm{O}$ & $\mathrm{H}_{2} \mathrm{O}^{+}$ & $\mathrm{CO}_{2}$ & - \\
\hline 4.9 & 0.45 & 0.25 & 55.4 & 0.1 & 0.68 & 1.56 & 0.43 & - \\
\hline
\end{tabular}

We used a mixture of scheelite concentrate and carbonaceous reducing agent for the experiment. In one case the mixture was preliminarily milled with the reducing agent, and in the other we used the mixture without pretreatment with particles sized approximately $500-800$ microns.

The experiments were conducted using the equipment with the output power density at the plasma burner between the cathode and anode reaching about $\mathrm{g}>10^{6}-10^{8} \mathrm{~W} / \mathrm{cm}^{2}$. Exposure was performed for 30-50 seconds. The process temperature of the synthesis was about 4-6 thousand ${ }^{\circ} \mathrm{C}$.

The reactor that received the mixture reacted in the plasma flow, and the burner, through which the mixture was fed, were tightly connected and the access of air to the reactor chamber was minimized. The entire process of synthesis proceeded in the atmosphere of inert argon gas. After melting the mixture and its removal from the plasma burner, all the material from the reactor chamber got through the tube into the reservoir with distilled water, where the resulting material in the form of reduced tungsten and tungsten monocarbide was produced in addition to the rest of the slag. Obtained particles of resulting compounds of $\mathrm{W}-\mathrm{W}_{\mathrm{n}} \mathrm{C}$ were sifted through a set of sieves, and represented samples of $0.2-0.5 \mathrm{~mm}$ in diameter (Figure 1).

\section{Results and discussions}

The proportion of $\mathrm{WC}$ in a dispersed material was about $58 \%$, while the content of tungsten reached about $21 \%$. The remaining approximately $11 \%$ of the material was presented by the slag. It is important to note that the proportion of the evaporated material was about $10 \%$ of the total amount of the entire portion taken for the experiment (1 portion of the mixture for the experiment was taken in the amount of $100 \mathrm{~g}$ ). Subsequently, during the same experiments under the same input parameters, the amount of evaporated material did not change.

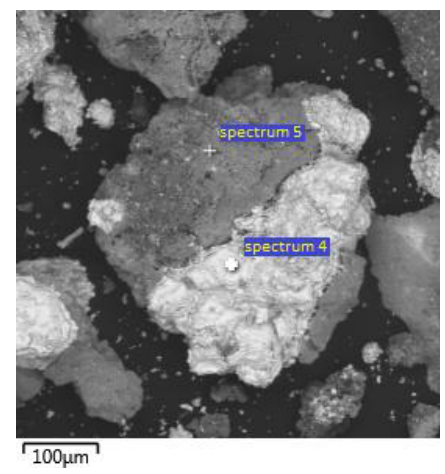

Fig. 1. The picture of tungsten carbide obtained using a scanning electron microscope VEGA 3 LMH. 
Table 2. The content of chemical elements in synthesized materials based on W/WC.

\begin{tabular}{|l|c|c|c|c|c|c|c|c|}
\hline $\begin{array}{l}\text { Chemical } \\
\text { element (wt.\%) }\end{array}$ & $\mathrm{C}$ & $\mathrm{O}$ & $\mathrm{Al}$ & $\mathrm{Si}$ & $\mathrm{P}$ & $\mathrm{S}$ & $\mathrm{Ca}$ & $\mathrm{W}$ \\
\hline Spectrum 5 & 4.82 & 37.86 & 0.94 & 1.19 & 0.27 & 0.57 & 53.83 & 0.52 \\
\hline Spectrum 4 & 7.9 & 0.8 & - & - & - & - & 0.01 & 92.1 \\
\hline
\end{tabular}

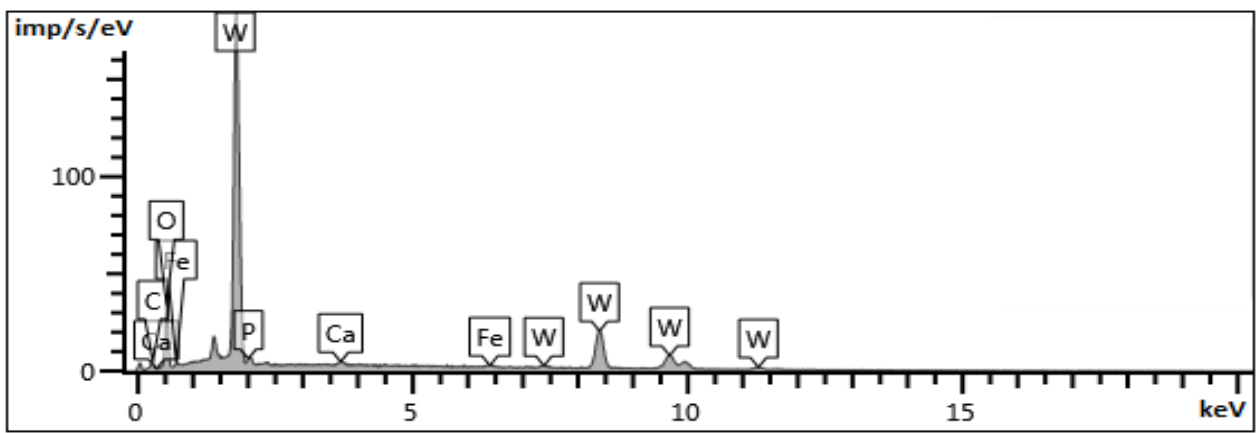

Fig. 2. Spectrogram of produced tungsten carbide after exposure to a high-density energy g $>10^{4}$ $10^{5} \mathrm{~W} / \mathrm{cm}^{2}, \mathrm{~g}>10^{6}-10^{8} \mathrm{~W} / \mathrm{cm}^{2}$.

Chemical analysis of the synthesized material showed the presence of $\mathrm{CaCO}_{3}, \mathrm{~W}_{2} \mathrm{C}$, $\mathrm{WC}$, carbon residue not involved in the reduction reaction and samples of tungsten in the form of fused particles.
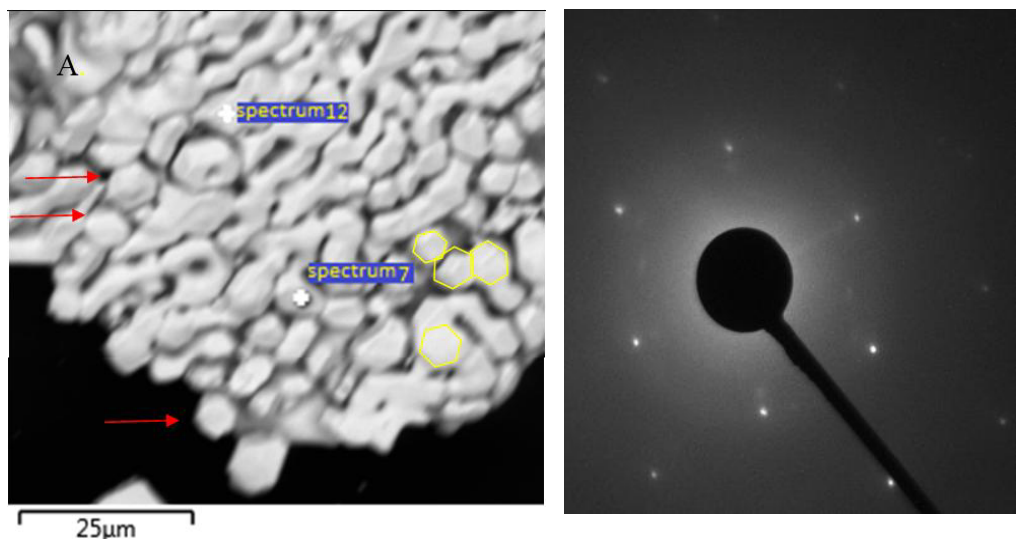

Fig.3. Fused tungsten carbide WC crystals of hexagonal structure. A - photo after «VEGA 3 LMH», B - scanning electron microscope.

Experiments performed with unprocessed concentrate have shown relatively similar results except for the increased proportion of resulting synthesized material $\mathrm{W}-\mathrm{W}_{\mathrm{n}} \mathrm{C}$. $\mathrm{We}$ can assume that synthesis proceeds much better with mechanical activation, aided by the reactions occurring at the time of grinding of the two components at the molecular level. Under such conditions the amount of produced material $\mathrm{W}-\mathrm{W}_{\mathrm{n}} \mathrm{C}$ is about $68-72 \%$. The type of the synthesized material after determination of its phase composition is WC with hexagonal structure of crystals. It is presented in Figure 3 after being washed with distilled water. The content of tungsten $\mathrm{W}$ was about $94-95 \%$ out of the total amount, and the content of carbon $\mathrm{C}$ was within the range of $6-5 \%$. 


\section{Conclusion}

1. The reduction of the tungsten carbide phase $\left(\mathrm{WC}, \mathrm{W}_{2} \mathrm{C}\right)$ from multi-component tungsten mineral resources by high-density energy about $\mathrm{g}>10^{4}-10^{5} \mathrm{~W} / \mathrm{cm}^{2}$ is possible and it significantly reduces the time for its synthesis;

2. The purity of the synthesized material reaches $92-95 \%$, which requires further study to increase the amount of tungsten carbide in the products of synthesis. Another possible prospect is the shift to producing carbon free tungsten and mineral resources;

3. the research has shown the prospects of employing high-density energy for the synthesis of refractory compounds from tungsten mineral resources, which will allow us to shift from the traditional forms of production;

4. During the preliminary mechanical activation of mixture components, high-temperature synthesis proceeds with the release of larger amounts of tungsten-containing synthesized products in the form of $\mathrm{WC}$ and $\mathrm{W}_{\mathrm{n}} \mathrm{C}$.

5. During the exposure to plasma the process temperature in the transition passage between the reactor and the burner reached about $5500 \mathrm{~K}$ and decreased with distance from the arcing zone to $3500 \mathrm{~K}$, which allowed us to use only carbon-containing material as a reducing agent. It is also necessary to consider that in the experiments carbon was added to mixture with $10 \%$ excess, but due to specific features of the synthesis process, this amount of carbon must be increased on account of the sublimation of carbon in the form of oxides $\mathrm{CO}$ and $\mathrm{CO}_{2}$.

\section{References}

1 E.N. Kuz'michev, A.D. Verkhoturov, D.S. Shtarev, Applied Mechanics and Materials 775, 165-169 (2015)

2 Y.N. Tumanov, Plazmennye, vysokochastotnye, mikrovolnovye i lazernye tehnologii v himiko-metallurgicheskih protsessah (FIZMALIT, Moscow, 2010)

3 S.A. Pegov, Vestnik RAN 84, 1082-1089 (2004)

4 A.D. Verkhoturov, E.G. Babenko, E.N. Kuzmichev, Journal of Advanced Materials 1, 75-78 (2003)

5 D. Chen, H. Wen, H. Zhai, H. Wang, X. Li, R. Zhang, J. Sun, L. Gao, J. Am. Ceram. Soc. 93(12), 3997-4000 (2010)

6 R. Koc, S.K. Kodamaka, T. Carbide, J. of the European Ceramic Society 20, 18591869 (2000)

7 M.F. Zawrah, J. Ceramics International 33, 155-161 (2007)

8 K.M. Reddy, T.N. Rao, J. Joardar, Materials chemistry and physics 128, 121-126 (2011)

9 G.A. Meerson, A.N. Zelikman, Mettalurgiya redkih metallov (Metallurgizdat, Moscow, 1973)

10 A.D. Verkhoturov, E.G. Babenko, E.N. Kuz'michev, Theoretical Foundations of Chemical Engineering September 48, 716-721 (2014)

11 Metal Powders: Technologies and Applications (Elsevier, 2009)

12 D.V. Onishchenko, Inorganic Materials 48, 1096-1101 (2012)

13 E. Hornbogen, Werkstoffe (Springer, Germany, 2006)

14 E. Hornbogen, G. Eggeler, E. Werner, Werkstoffe (Springer, Berlin, 2012) 